\title{
Research on Artificial Fish Swarm Algorithm with Cultural Evolution for Subcarrier Allocation
}

\author{
LIU Mingzhu, LI Xin, ZHANG Mingyu and LI Chang \\ The Higher Educational Key Laboratory for Measuring \& Control Technology \\ and Instrumentations of Heilongjiang Province \\ School of Measurement and Control Technology and Communications \\ Engineering \\ Harbin University of Science and Technology, Harbin, China, 150080 \\ lmz@hrbust.edu.cn
}

\begin{abstract}
In the resource allocation process of multi-user OFDM system, in order to realize the purpose of maximizing the total transmission rate under constant power, a new subcarrier allocation algorithm has been proposed in this paper, which is to introduce cultural evolution method into the original artificial fish swarm algorithm. Because the population space in cultural algorithm framework has the advantage of guiding search process, the new algorithm can effectively overcome the defect of falling into local extreme which generally exists in the fish swarm algorithm in the resource allocation. At the same time, the proposed new algorithm also can make it easy to quantify the optimized goals and variable values. Simulation results show that the proposed artificial fish swarm algorithm with cultural evolution (CE-AFS) has been greatly improved the global search ability and convergence speed compared with the AFS algorithm and Shen algorithm.
\end{abstract}

Keywords: OFDM, subcarrier allocation, artificial fish swarm algorithm, cultural evolution, global optimization

\section{Introduction}

The technology of orthogonal frequency division multiplexing (OFDM) is a multicarrier digital modulation technique which can improve the utilization rate of wireless network channel [1]. The technology can reduce the inter-carrier interference and dynamically allocate channel bandwidth. Resource allocation in OFDM system is an important ways to guarantee service quality and improve system capacity, and it has been the hot research field in communication engineering [2]. At present, according to the different optimization objectives, the adaptive resource allocation algorithm of OFDM system can be divided into two categories. One is to maximize the transmission rate with a certain bit error rate when the total power is constrained, which has been called rate adaptive rule (RA rule) [3]. The other is to make the transmit power minimization in a certain system transmission rate and bit error rate, which has been called power adaptive rule (PA rule) [4].

Shen algorithm and artificial fish swarm algorithm (AFS) both belong to the rate adaptive optimization algorithm. Shen algorithm is the method to maximize the system total transmission rate under the fixed rate ratio condition for all users. But Shen algorithm needs a large amount of calculation in the allocation process, and it also can not obtain the satisfactory total transmission rate in the system [5]. AFS algorithm had been introduced in reference [6], it improved the system transmission rate in a certain extent, but AFS algorithm was easy to get into the trouble of local extremum value because of the 
slow convergence speed in the later operation, and there was a certain probability can not find the optimal subcarrier allocation scheme [7]. Because the cultural algorithm has strong searching ability for optimal problems, it will be introduced into the AFS algorithm to improve the system performance. Therefore, a new optimization algorithm for subcarrier allocation has been proposed under the RA rule, which is cultural evolution artificial fish swarm algorithm (CE-AFS). The new algorithm has good searching ability, and it can effectively solve the low efficiency searching problem of original adaptive algorithm in the subcarrier allocation process.

\section{Resource Allocation Models of OFDM System}

Let $N$ be the number of subcarriers in OFDM system, $K$ be the number of users, $B$ denote system total bandwidth and $P$ be the total power. Assume that the channel is frequency selective fading channel, subcarriers are independent with each other, and each subcarrier can only correspond to one user simultaneously. Let $\rho_{k n}$ be the subcarrier allocation factor, if $n$-th subcarrier has been allocated to the $k$-th user, then $\rho_{k n}=1$, or else $\rho_{k n}=0$. When $\rho_{k n}=1$, let $h_{k n}$ be the instantaneous channel gain, and $P_{k n}$ be the average power obtained from $n$-th subcarrier by the $k$-th user. The transmission rate of the $k$-th user on the $n$-th subcarrier can be obtained by the following formula (1).

$$
R_{k n}=\rho_{k n}\left(\frac{B}{N}\right) \log _{2}\left[1+\frac{P_{k n} h_{k n}^{2}}{I_{k n}+N_{0} \frac{B}{N}}\right]
$$

In formula (1), $I_{k n}$ is the interference power which produced by the Doppler frequency shift on the $n$-th subcarrier to the $k$-th user, and $N_{0}$ is the white Gaussian noise power spectral density. Thus, the total transmission rate of the $k$-th user can be expressed as the following formula (2).

$$
R_{k}=\sum_{n=1}^{N} \rho_{i n}\left(\frac{B}{N}\right) \log _{2}\left[1+\frac{P_{k n} h_{k n}^{2}}{I_{k n}+N_{0} \frac{B}{N}}\right]
$$

The total transmission rate $R$ for all users has been shown in formula (3), which is based on RA rule.

$$
R=\max \sum_{k=1}^{K} \sum_{n=1}^{N} \rho_{k n}\left(\frac{B}{N}\right) \log _{2}\left[1+\frac{P_{k n} h_{k n}^{2}}{I_{k n}+N_{0} \frac{B}{N}}\right]
$$

In fact, there are some constraint conditions in formula (3), which have been shown in expression (4).

$$
\begin{aligned}
& \sum_{k=1}^{K} \rho_{k n}=1, \quad \sum_{k=1}^{K} \sum_{n=1}^{N} \rho_{k n}=N \\
& \sum_{n=1}^{N} P_{k n} \leq P, \quad P_{k n} \geq 0
\end{aligned}
$$

From formula (3), it can be seen that if average power $P_{k n}$ remains constant, in order to increase the total transmission rate $R$, the channel gain $h_{k n}$ can be amended and improved through some reasonable subcarrier allocation method. It means to maximize the transmission rate, a new subcarrier allocation algorithm can be used, and that is CE-AFS algorithm. Because cultural evolution algorithm has the character of good optimization ability, it can be applied into the original AFS algorithm to overcome the defect of slow convergence rate and optimize the subcarrier allocation in OFDM system. 


\section{Fundamental of CE-AFS Algorithm}

\subsection{Original Artificial Fish Swarm Algorithm}

The fundamental of artificial fish swarm algorithm is to simulate and study the fish' behaviors of preying, swarming, chasing and cooperating with each other in natural environment, and then to construct some mathematical models to find the optimal values ${ }^{[8][9]}$. These mathematic models have been described in the following.

Assume that there are $N$ artificial fishes constituting a swarm in a target researching area, the status of the $N$ artificial fishes can be expressed by vector $\mathbf{X}=\left(X_{1}, X_{2}, \cdots, X_{N}\right)$ and the element $X_{i}(\mathrm{i}=1,2, \cdots, N)$ in $\mathbf{X}$ is the expected optimization variable for every artificial fish. Let vector $\mathbf{Y}=f(\mathbf{X})$ be the food density where the artificial fishes located. In the AFS algorithm, $\mathbf{Y}$ is the value of the objective function, and the behavior of each single fish will be determined by vector $\mathbf{Y}$.

3.1.1. Preying Behavior Model: Assume that the current status of the $i$-th artificial fish has been denoted by $X_{i}$, and its food density is $Y_{i}$. Selecting a status $X_{j}$ randomly in $i$-th artificial fish perception range, and its food density has been denoted by $Y_{j}$. If $Y_{j}>Y_{i}$, the artificial fishes will make a step forward to $X_{j}$, else the model will select other status $X_{q}$ randomly, and then determine whether the moving condition has been satisfied. Let trynumber be the maximum number of tests, and if the moving condition still can not be satisfied when the test number reaches to trynumber by the above step, the $i$-th artificial fish will move one step randomly.

The preying behavior can be expressed by function prey which has been shown in formula (5).

$$
\operatorname{prey}\left(X_{i}\right)=X_{i}+\operatorname{Rand}() \cdot \operatorname{step} \cdot \frac{X_{j}-X_{i}}{\left\|X_{j}-X_{i}\right\|}
$$

where, $\boldsymbol{R a n d}()$ is a random number between 0 to 1 , and variable step is the preset step size.

3.1.2. Swarming Behavior Model: Let $n_{f}$ be the number of artificial fishes which are in the perception domain of $i$-th fish which has been denoted by $X_{i}$, and $Y_{c}$ be the food density in the center position $X_{c}$. Let $\delta$ be the preset crowded degree factor, which can control the swarm density and avoid overcrowding of artificial fishes. If $Y_{i}<Y_{c}$ and $\frac{n_{f}}{N}<\delta$ have been satisfied at the same time, it means that the center of fish swarm is not crowded, and there is enough food in the center position, and the artificial fishes will move one step toward the center position $X_{c}$, else do the preying behavior shown in the above. The swarming behavior can be indicated by function swarm which has been shown in formula (6).

$$
\operatorname{swarm}\left(X_{i}\right)=X_{i}+\operatorname{Rand}() \cdot \operatorname{step} \cdot \frac{X_{c}-X_{i}}{\left\|X_{c}-X_{i}\right\|}
$$

3.1.3. Chasing Behavior Model: If a fish has found plenty of food, other fishes will follow it, this is chasing behavior. By searching the adjacent areas, the artificial fish $X_{\max }$ with maximum food density will be found and the food density will be denoted by $Y_{\max }$. If $Y_{i}<Y_{\max }$ and $\frac{n_{f}}{N}<\delta$, then the searching will move one step toward the direction of the maximum objection function value, else do preying behavior as the above. Chasing behavior can be described by the function chase shown in formula (7). 


$$
\operatorname{chase}\left(X_{i}\right)=X_{i}+\operatorname{Rand}() \cdot \operatorname{step} \cdot \frac{X_{\text {max }}-X_{i}}{\left\|X_{\text {max }}-X_{i}\right\|}
$$

3.1.4. Bulletin Board: Bulletin board is used to record the optimal status of the single artificial fish. Every artificial fish updates its own position constantly according to the three behaviors shown in the above, and then compares its current status with bulletin board. If its own status is superior to the current status in bulletin board, the bulletin board will be rewritten with the new artificial fishes' status. In this way, it can ensure that the optimal status will always be recorded in the bulletin board.

\subsection{Cultural Algorithm}

Cultural algorithm was proposed firstly by Reynolds in 1994 [10]. The main idea of this algorithm is to simulate the procedure of cultural evolution and to get the knowledge for solving some problem from population evolution process. This knowledge is also called 'belief', which will be used to guide the search process. The general framework model of cultural algorithm has been given in the Figure 1. From the Figure 1, the structure of cultural algorithm is composed of two parts, one is the population space, and the other is belief space.

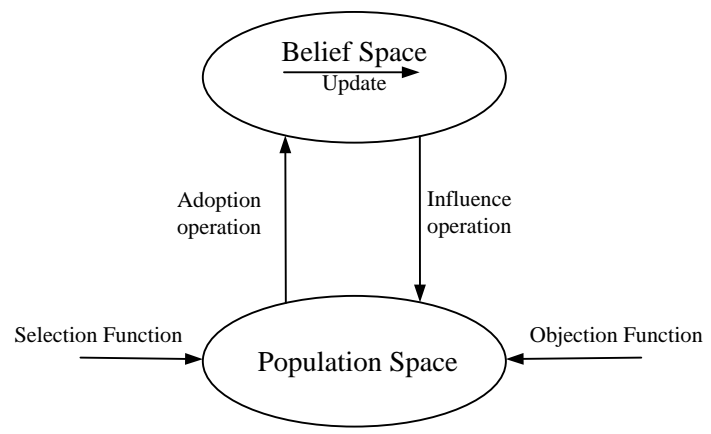

Figure 1. Basic Framework of Cultural Algorithm

Generally, elite individuals in the lower population space have been contributed to the upper belief space, and the belief space also evolves its own elites individuals, which will influence or control the population space in turn. Because of the double evolution structure features of cultural algorithm, it makes the swarm be able to evolve with a fast speed, and show the better global optimization performance in searching process $[11,12]$. In the lower population space, the individual will firstly evolve according with belief knowledge rules. According to evolutionary situation, the elite individual information will be extracted by adoption operation, and then update the status in the belief space. The population evolution will take on by influence operation according to the extracted belief knowledge, thus the population space and belief space will be interacted with each other [13]. In the cultural algorithm, a kind of multi-evolution computational model has been provided, and any evolution processes which meet the requirement of cultural algorithm can be implanted into the cultural algorithm structure. That is the cultural evolution process in population and belief spaces.

\section{Subcarrier Allocation Algorithm with CE-AFS}

The main idea of the CE-AFS algorithm is to make use of the structure model of cultural algorithm to establish the main lower population space of artificial fish swarm and upper belief upper space, it will make original artificial fish swarm form the convective searching character [14]. The global optimal solution will be obtained by updating the bulletin board and objection function values in the process of the improved 
artificial fish evolution. This double circular evolution structure comes from adoption operation and influence operation between the artificial fish population space and belief space, which will keep the acceptable knowledge and abandon unacceptable knowledge, and then obtain the best global search value and searching performance [15].

CE-AFS algorithm has been introduced to solve adaptive subcarrier allocation problem in OFDM system. The generated random vectors have been set as the initial scheme of subcarrier allocation, and the objective function is the obtained maximum transmission rate of the system which has been shown in formula (3). Through iterative operation, an optimal subcarrier allocation scheme can be obtained. By the iterative operation, it can get the maximum value of the objective function and maximize the total transmission rate of OFDM system. The implementation steps of CE-AFS algorithm have been shown in the following.

Step (1): Subcarrier Pre-allocation. Assume that $K$ is the number of users in OFDM system, $N$ is the number of subcarriers which are distributed in the whole system frequency band. Let $M$ be the per-set population scale. Use $N M$ - dimension random vectors to express the initial artificial fish swarm, and the elements in the vector is a number between 1 to $N$. In order to ensure the fairness among the users, the number of subcarriers which had been allocated to each user should be proportional to the bit number which the user transmitted, that is $M_{1}: M_{2}: \cdots: M_{K}=R_{1}: R_{2}: \cdots: R_{K}$. The elements $1 \sim M_{1}$ should be assigned to the first user, and the elements $\left(M_{1}+1\right) \sim\left(M_{1}+M_{2}\right)$ should be assigned to the second user, and so on, until all $N$ elements have been assigned to all users. For example, assume that the vector is $[3,9,1,5,6,10,2,8,31,19,7, \cdots]$, if the number of subcarriers allocated to the $1^{\text {st }}$ user is $M_{1}$, and $M_{1}=4$, it means that the subcarriers $3,9,1,5$ are assigned to the $1^{\text {st }}$ user. In the same way, if $M_{2}=5$, it means that the subcarriers $6,10,2,8,31$ are assigned to the $2^{\text {nd }}$ user, and so on. When $\sum_{k=1}^{K} M_{k}<M$, the rest subcarriers are allocated to the users in good channel condition, until all the subcarriers are assigned completely.

Step (2): Space Initialization. Firstly, determine the population space of CEAFS algorithm. According to the subcarrier allocation method shown in step1, $N$ artificial fish individuals will be generated randomly, and then form the initial artificial fish swarm. Each artificial fish individual represents a subcarrier allocation scheme. After setting the parameters of step size step and crowded degree factor $\delta$, calculate and compare the fitness values for each artificial fish in the initial swarm, and then select the optimal status and assign the updating prey, swarm and chase function values into the bulletin board. Secondly, build the belief space of CE-AFS algorithm. The process of subcarrier allocation in belief space is same as the one in the population space. However, the difference is that the scale of the elite individuals in belief space should be only $30 \%$ of the total population scale.

Step (3): Adoption Operation. For the cultural evolution process in population space, let acceptstep be the running time of adoption operation, and use the individual with the maximum current global objective function value in population space to replace the one with minimum objective function value in belief space.

Step (4): Influence Operation. In the cultural evolution process of belief space, let Influencestep be the running time of influence operation, and use the individuals with superior adaptive values in belief space to replace those unfit adaptive values individuals in population space. The replacement process has been shown in formula (8).

$$
\text { Inflencestep }=Q_{1}+\frac{\text { endstep }- \text { currentstep }}{\text { endstep }} \times Q_{2}
$$

where endstep is the preset maximum evolution generation value of artificial fish swarm, and currentstep is the current evolution generation value. $Q_{1}$ and $Q_{2}$ are 
constants, and those will be set to 15 and 100 respectively according to reference [14].

In the initial stage of cultural evolution algorithm, the belief space has a slight effect on the population space. Calculate the objective function value of each artificial fish, and then compare with these values of other artificial fish. According to the objective function value, each artificial fish will choose whether to perform the preying, swarming or chasing behavior. Meanwhile, the artificial fishes constantly update their locations and the optimal status is always recorded on the bulletin board.

Step (5): The Global Optimal Value Selection. At the end of each iteration operation, the objective function value of each artificial fish will be compared with the value on the bulletin board. If the objective function value is superior to the one on bulletin board, then the values on bulletin board will be replaced by the current objective function value, otherwise, the values on bulletin board will remain unchanged. When the iteration time reaches to the given maximum value, the global optimal value selection process will be terminated, otherwise, repeat the above steps (3-5) until the termination condition has been satisfied.

Step (6): Result Output. The final record on the bulletin board is the global optimal value, and the corresponding output is the required optimal subcarrier allocation scheme.

\section{Simulation and Result Analysis}

To test the proposed CE-AFS algorithm performance, the total transmission rate and average transmission rate at different number of users by CE-AFS algorithm have been analyzed in the following, and these properties have also been compared with those by original AFS algorithm and Shen algorithm which bases on proportional fairness criterion respectively. The simulation parameters have been set as follows.

Assume that the channel is Rayleigh fading channel, the maximum Doppler frequency shift is $15 \mathrm{~Hz}$, and the power spectral density of AGWN is $-80 \mathrm{dBW} / \mathrm{Hz}$. Let the frequency bandwidth of OFDM system be $1 \mathrm{MHz}$, and the total transmission power is $1 \mathrm{~W}$. Set the maximum iteration time is 160 , trynumber is 5, acceptstep is 10 , and the step size step $=2$. Assume that the population size is $M=30$, and crowded degree factor $\delta=0.2$.

When the number of subcarriers is $64,128,256$ respectively, the computation time of AFS and CE-AFS algorithms has been listed in the table1. From table1, it can be found that the average computation time of CE-AFS algorithm is less than the one of AFS algorithm when the number of subcarrier is same, it means that computation complexity of the proposed CE-AFS algorithm has been reduced observably. The total transmission rate curves of Shen, AFS and CE-AFS algorithms in 4-user OFDM system have been shown in figure 2 with the same transmitting bit information.

Table 1. Computation Time Comparison between Afs and Ce-Afs Algorithms

\begin{tabular}{ccccccc}
\hline \multicolumn{2}{c}{$N=64$} & \multicolumn{2}{c}{$N=128$} & \multicolumn{2}{c}{$N=256$} \\
\hline $\begin{array}{c}\mathrm{N}_{\mathrm{O}} \\
\mathrm{AFS} / \mathrm{ms}\end{array}$ & AFS/ms & CE-AFS/ms & AFS/ms & CE-AFS/ms & AFS/ms & CE- \\
\hline 1 & 24.341 & 9.452 & 42.547 & 19.464 & 71.852 & 38.987 \\
2 & 22.158 & 10.589 & 40.331 & 17.652 & 75.871 & 35.468 \\
3 & 20.229 & 11.115 & 45.875 & 21.776 & 69.421 & 34.898 \\
4 & 19.889 & 9.332 & 47.221 & 16.252 & 74.763 & 37.284 \\
5 & 17.775 & 12.479 & 49.436 & 20.023 & 76.447 & 40.565 \\
6 & 26.545 & 8.226 & 44.769 & 21.338 & 73.495 & 33.343 \\
7 & 17.982 & 14.692 & 41.548 & 22.325 & 78.692 & 43.562
\end{tabular}




\begin{tabular}{ccccccc}
8 & 25.693 & 10.448 & 47.884 & 24.885 & 70.226 & 41.853 \\
9 & 18.464 & 15.231 & 43.625 & 20.764 & 77.962 & 35.794 \\
10 & 19.539 & 8.221 & 50.256 & 25.683 & 79.641 & 36.679 \\
\hline
\end{tabular}

AFS iteration time: $N=64,21.2615 \mathrm{~ms} ; N=128,45.3519 \mathrm{~ms} ; \quad N=256,74.8370 \mathrm{~ms}$ CE-AFS iteration time: $N=64,10.9785 \mathrm{~ms} ; \quad N=128,21.0162 \mathrm{~ms} ; \quad N=256,37.8433 \mathrm{~ms}$

From the Figure 2, it can be seen that the three algorithms all can find the optimal value within 80 generations, and the CE-AFS algorithm has the fastest searching speed, however, when increasing the iteration times, it can be found that the differences of searching speed is not obvious with the three algorithms.

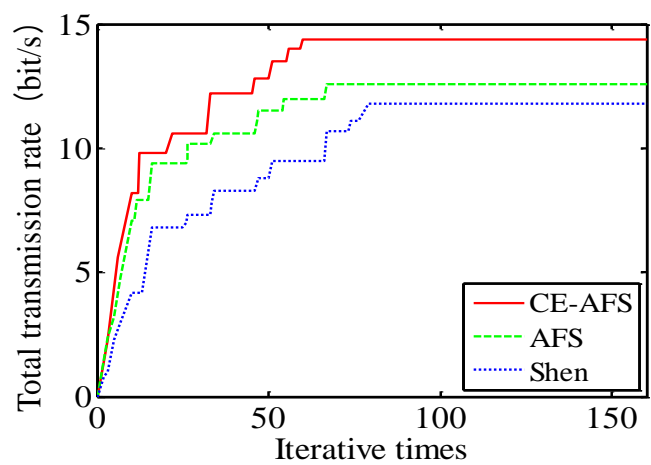

Figure 2. Relationship Curve of Iteration Times and Total Transmission Rate $(N=128, K=4)$

Figure 3 has shown the comparison charts of total transmission rate by the three algorithms in the case of 8 users. The variation trend of total transmission rates is similar to that in Figure 2. From the Figure 2 and Figure 3, it can be found that both CE-AFS and AFS algorithms are better than Shen algorithm in searching speeds, and the searching process of CE-AFS algorithm has been completed within 70 generations, it is faster than Shen algorithm which is nearly to 120 generations. Because of the introduction of cultural evolution method into original AFS algorithm, the CE-AFS algorithm has excellent convergence speed and obtains the global optimal value with the least number of iterations, especially in the large number of users system. Moreover, under the condition of the fixed number of users and iteration times, the total transmission rate of the CE-AFS algorithm is the largest in the three algorithms, and it also conforms to the RA rule.

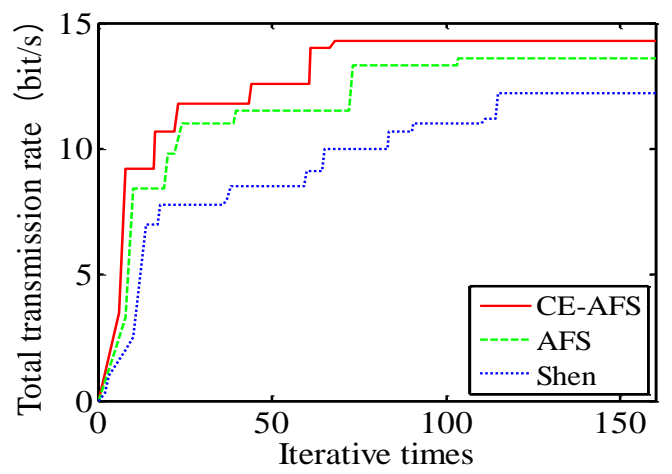

Figure 3. Relation Curve of Iteration Times and Total Transmission Rate $(N=128, K=8)$ 
With the different number of users, compare the average transmission rates of each user of the three algorithms in the figure 4 when the maximum iteration time is 160. From the Figure 4, it can be seen that with the increase of the number of users, the average transmission rate of each user has been declined. Regardless of the number of users is 4,8 , or 12 , the average transmission rate of each user in CE-AFS algorithm is the highest compared with the other two algorithms, thus, it means that the searching ability of CE-AFS algorithm is superior to the other two methods. The advantage of quick search and convergence capability of CE-AFS algorithm is very obvious, especially in the large of number of users OFDM system. For example, the average transmission rate of each user in CE-AFS algorithm compared with Shen algorithm is increased about $10.1 \%$ when the number of users is 4 , and in the same way, it is increased about $19.3 \%$ and $51.6 \%$ when the number of users is 8 and 12 respectively.

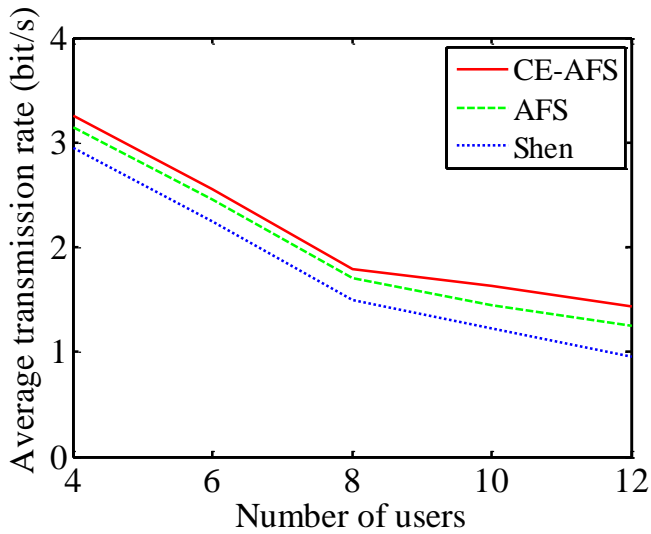

Figure 4. Comparison Curves on Average Transmission Rate In Different Number of Users System

\section{Conclusion}

A new CE-AFS algorithm has been proposed in this paper, which introduces the cultural evolution method into traditional AFS algorithm. It benefits to improve the global search ability of original AFS algorithm for the subcarrier allocation in multi-user OFDM system. Simulation results show that CE-AFS algorithm has faster convergence speed and stronger global search ability, and at the same time, it only needs fewer iteration times to get optimal subcarrier allocation scheme compared with original AFS algorithm and Shen algorithm, and it also can improve the total transmission rate.

\section{ACKNOWLEDGEMENTS}

This paper is supported by Natural Science Fund of China (61179024); Scientific Research Fund of Heilongjiang Provincial Education Department (12521087); Harbin Science and Technology Innovation Talents Fund (2009RFQXG075).

\section{References}

[1] Z. Huang, X. Zhao and C. He, "Fast optimal resource allocation algorithm for multicast OFDM systems", Wireless Communications \& Signal Processing (WCSP), International Conference, (2012) October 25-27, pp. 1-5, Huangshan, China.

[2] D. Kim, T. Fujii and K. Lee, "A resource allocation algorithm for OFDM-based cellular system serving unicast and multicast services", EURASIP Journal on Wireless Communications and Networking, vol. 1, (2013), pp. 41. 
[3] W. Rhee and J. M. Cioffi, "Increase in capacity of multiuser OFDM system using dynamic sub-channel allocation", Proc of Vehicular Technology Conference, (2000), May 15-18, pp. 1085-1089 Tokyo, Japan.

[4] C. Y. Wong, R. S. Chen and K. B. Letaief, "Multiuser OFDM with adaptive subcarrier bit and power allocation", IEEE Journal on Selected Areas in Communications, vol. 10, no. 17, (1999), pp. 1747-1758.

[5] K. S. Shen, J. G. Andrews and B. L. Evans, "Adaptive resource allocation in multiuser OFDM systems with proportional rate constraints", IEEE ransactions on wireless communications, vol. 4, no. 6, (2005), pp. 2726-2737.

[6] O. Ender and C. K. Mohan, "Analysis of a simple particle swarm optimization system", Proceedings of the 1998 Artificial Networks in Engineering Conference, (1998) November 1-4, pp. 253-258 St. Louis, MO, USA.

[7] L. P. Wang, G. F. Sun and Y. M. Chen, "Dynamic cross-layer resource allocation in multiuser OFDM system based on artificial fish swarm algorithm", Journal of Circuits and Systems, vol. 3, no. 16, (2011), pp. 87-91.

[8] Z. YiKui, X. Ying and G. J. Ying, "A novel artificial fish swarm algorithm based on multi-objective optimization", $8^{\text {th }}$ International Conference on Intelligent Computing Theories and Applications, (2012) July 25-29, pp. 67-73, Huangshan, China.

[9] W. Guo, G. Fang and X. Huang, „An improved chaotic artificial fish swarm algorithm and its application in optimizing cascade hydropower stations", Business Management and Electronic Information (BMEI), (2011) May 13-15, pp. 217-220, Guangzhou, China.

[10] R. G. Reynolds and S. William, "Problem solving using cultural algorithms", Proceedings of the $1^{\text {st }}$ IEEE Conference on Evolutionary Computation, (1994) June 27-29, pp. 131-139, Orlando, USA.

[11] H. Y. Lan, X. Y. Yang and H. B. Liu, "Resource allocation for multiuser OFDM system based on cultural algorithm", Journal of Jilin University (Engineering and Technology Edition), vol. 3, no. 41, (2011), pp. 226-230.

[12] W. Zhou and Y. Bu, "Cultural particle swarm optimization algorithm and its application", $24^{\text {th }}$ Control and Decision Conference, (2012) May 23-25, pp. 740-744, Taiyuan, China.

[13] M. Hai and W. Yanjiang, "Cultural algorithm based on particle swarm optimization for function optimization", $5^{\text {th }}$ International Conference on Natural Computation, (2009) August 14-16, pp. 224-228, Tianjian, China.

[14] L. Z. Liu and Y. Q. Zhou, "Hybrid global optimization algorithm based on artificial fish swarm and cultural algorithm", Application Research of Computer, vol. 12, no. 26, (2009), pp. 4446-4448.

[15] X. J. Bi and W. W. Chao, "Adaptive Sub-Carrier allocation for an orthogonal frequency division multiple access system based on a particle swarm optimization algorithm", Journal of Harbin Engineering University, vol. 8, no. 31, (2010), pp. 1100-1104. 
International Journal of Hybrid Information Technology

Vol.8, No.6 (2015) 